PROCEEDINGS OF THE

AMERICAN MATHEMATICAL SOCIETY

Volume 125, Number 9, September 1997, Pages 2513-2520

S 0002-9939(97)03847-1

\title{
EVERY LOCAL RING IS DOMINATED BY A ONE-DIMENSIONAL LOCAL RING
}

\author{
ROBERT GILMER AND WILLIAM HEINZER
}

(Communicated by Wolmer Vasconcelos)

\begin{abstract}
Let $(R, \mathbf{m})$ be a local (Noetherian) ring. The main result of this paper asserts the existence of a local extension ring $S$ of $R$ such that (i) $S$ dominates $R$, (ii) the residue field of $S$ is a finite purely transcendental extension of $R / \mathbf{m}$, (iii) every associated prime of (0) in $S$ contracts in $R$ to an associated prime of (0), and (iv) $\operatorname{dim}(S) \leq 1$. In addition, it is shown that $S$ can be obtained so that either $\mathbf{m} S$ is the maximal ideal of $S$ or $S$ is a localization of a finitely generated $R$-algebra.
\end{abstract}

\section{INTRODUCTION}

Let $R$ be a commutative ring with identity having a unique maximal ideal $\mathbf{m}$. We write in this situation that $(R, \mathbf{m})$ is a quasilocal ring. A quasilocal extension ring $(S, \mathbf{n})$ of $R$ is said to dominate $R$ if $\mathbf{m} \subseteq \mathbf{n}$ or, equivalently, if $\mathbf{n} \cap R=\mathbf{m}$.

There are several well-known results concerning domination in the study of local rings and quasilocal rings. For example:

(1) Every quasilocal domain is dominated by a valuation domain [N, (11.9)].

(2) If $(R, \mathbf{m})$ is a quasilocal domain and $F$ is a subfield of the field of fractions of $R$, then $R \cap F$ is a quasilocal domain and $R$ dominates $R \cap F$.

(3) Every local ring is dominated by a complete local ring [N, (17.6)].

(4) (Chevalley) Every local domain is dominated by a rank-one discrete valuation domain (DVR) [Ch, page 26]. (For a generalization of (4), see [CHL].)

Concerning Result (4), the proof Chevalley gives for this also shows that a dominating DVR $V$ of the local domain $(R, \mathbf{m})$ can be taken inside the field of fractions of $R$, and, in view of the fact that residue extensions are finite algebraic when passing to the integral closure of a Noetherian domain [N, (33.10)], $V$ can be chosen so that the residue field of $V$ as an extension field of $R / \mathbf{m}$ is a finitely generated field extension. It is often the case that the residue field of $V$ is necessarily transcendental as an extension of $R / \mathbf{m}$, for if $R$ is complete and the residue field of $V$ is finite algebraic over $R / \mathbf{m}$, then by a result of Cohen [N, (30.6)], $V$ is a finitely generated $R$-module and therefore $\operatorname{dim}(R)=1$.

Since a DVR is a one-dimensional normal local domain (and conversely), a consequence of Result (4) is that a local domain is dominated by a one-dimensional local domain.

Received by the editors August 4, 1995 and, in revised form, March 12, 1996.

1991 Mathematics Subject Classification. Primary 13B02, 13C15, 13E05, 13 H99.

(C)1997 American Mathematical Society 
The main result of this paper is a ring-theoretic version of this last fact. If $(R, \mathbf{m})$ is a local (Noetherian) ring, we establish in Theorems 3.1 and 3.7 the existence of a local extension ring $S$ of $R$ such that:

(1) $S$ dominates $R$,

(2) the residue field of $S$ is a finite purely transcendental extension field of $R / \mathbf{m}$,

(3) every associated prime of (0) in $S$ contracts in $R$ to an associated prime of (0), so every regular element of $R$ remains regular in $S$, and

(4) $\operatorname{dim}(S) \leq 1$, and $\operatorname{dim}(S)=1$ unless $\operatorname{dim}(R)=0$.

In Theorem 3.1 we obtain an $S$ satisfying the conditions above that also has the property that $\mathbf{m} S$ is the maximal ideal of $S$, while in Theorem 3.7 we obtain an $S$ satisfying (1)-(4) that is a localization of a finitely generated $R$-algebra. ${ }^{1}$

We were originally motivated to consider the domination question of this paper by our work on the embedding of commutative rings in finite-dimensional rings [GH5]. For a local or quasilocal ring, a condition weaker than domination is that of having an extension ring of a certain form. It is well known that every Noetherian ring is a subring of an Artinian ring (see, for example, [GH2, (2.6)]). However, a local (Noetherian) ring having more than one associated prime is not a subring of a local Artinian ring. Our main result implies that every local ring is a subring of a one-dimensional local ring. There is no analogue to this result for arbitrary commutative rings in that [GH5, Example 1.6] establishes the existence, for each positive integer $n$, of a quasilocal ring $R_{n}$ of dimension $n$ that is not a subring of a ring of dimension less than $n$.

All rings considered in this paper are assumed to be commutative and unitary. If $R$ is a subring of a ring $S$, we assume that the unity of $S$ is contained in $R$, and hence is the unity of $R$. A nonzero element $r \in R$ is a regular element of $R$ if $r$ is not a zero divisor in $R$. All allusions to the dimension, $\operatorname{dim}(R)$, of a ring $R$ refer to its Krull dimension. Thus $\operatorname{dim}(R)=n$ if there is a chain $P_{0}<P_{1}<\cdots<P_{n}$ of prime ideals of $\operatorname{Spec}(R)$ and no chain of longer length.

\section{Ideals of $R$ CONTRACTED From the EXtension Ring $R(t)$}

(2.1). Let $(R, \mathbf{m})$ be a local ring, let $t$ be an indeterminate over $R$, and let $R(t)=R[t]_{\mathbf{m}[t]}$, the localization of the polynomial ring $R[t]$ at the multiplicative system of polynomials in $R[t]$ having a unit coefficient. It is clear that $R(t)$ is a local ring dominating $R$ with maximal ideal $\mathbf{m} R(t)$ and residue field isomorphic to $(R / \mathbf{m})(t)$, a simple transcendental extension of the residue field $R / \mathbf{m}$ of $R$. It is known [N, pages 17-18], [G, (33.1)], [GH1] that each ideal of $R$ is the contraction of its extension to $R(t)$. Since $R$ is Noetherian, it follows from the altitude theorem of Krull [N, (9.3)] that $\operatorname{dim}(R)=\operatorname{dim}(R(t))$. More generally, if $n>1$ is a positive integer and $t_{1}, \ldots, t_{n}$ are indeterminates over $R$, the ring $R\left(t_{1}, \ldots, t_{n}\right)=R\left[t_{1}, \ldots, t_{n}\right]_{\mathbf{m}\left[t_{1}, \ldots, t_{n}\right]}$ is a local ring isomorphic to $R\left(t_{1}\right)\left(t_{2}, \ldots, t_{n}\right)$ with residue field $R\left(t_{1}, \ldots, t_{n}\right) / \mathbf{m} R\left(t_{1}, \ldots, t_{n}\right)$, a pure transcendental extension of $R / \mathbf{m}$ in $n$ indeterminates; each ideal of $R$ is the contraction of its extension to $R\left(t_{1}, \ldots, t_{n}\right)$, and $\operatorname{dim}(R)=\operatorname{dim}\left(R\left(t_{1}, \ldots, t_{n}\right)\right)$.

\footnotetext{
${ }^{1}$ As suggested by the referee, it would be interesting to know more about the structure of one-dimensional local rings $S$ dominating a given $R$. One restriction on $S$ is that it must have at least as many minimal prime ideals as does $R$ (cf. [GH4, (2.1)]).
} 
(2.2). With $(R, \mathbf{m})$ and $t$ as in (2.1), suppose $a, b \in \mathbf{m}$, with $b$ a regular element of $R$. Let $\phi: R[t] \rightarrow R[a / b]$ denote the $R$-algebra homomorphism into the total quotient ring of $R$ such that $\phi(t)=a / b$. Assume that $\operatorname{ker} \phi \subseteq \mathbf{m}[t]$. Then $\mathbf{m} R[a / b]$ is a nonmaximal prime ideal of $R[a / b]$, and $\phi$ extends to a homomorphism of $R(t)$ onto $R[a / b]_{\mathbf{m} R[a / b]}$ such that $R(t) /(\operatorname{ker} \phi) R(t) \cong R[a / b]_{\mathbf{m} R[a / b]}$.

Lemma 2.3. Suppose $D$ is a Noetherian domain, $a, b \in D$ are such that the ideal $(a, b) D$ has height two, and $P$ is a prime ideal of $D$ with $(a, b) D \subseteq P$. Let $t$ be an indeterminate over $D$ and let $\phi: D[t] \rightarrow D[a / b]$ be the surjective $D$-algebra homomorphism such that $\phi(t)=a / b$. Then $\operatorname{ker} \phi \subseteq P[t]$.

Proof. The result follows for every prime ideal containing $(a, b) D$ provided it is true for each minimal prime of the ideal, so we may assume that $P$ is a minimal prime of $(a, b) D$, and is therefore of height two. Let $R=D_{P}$ and let $\mathbf{m}=P D_{P}$. Then $R$ is a 2-dimensional local domain and $(a, b) R$ is $\mathbf{m}$-primary. Let $R^{\prime}$ denote the integral closure of $R$, let $P^{\prime}$ be a maximal ideal of $R^{\prime}$ of height two, and let $R^{*}=R_{P^{\prime}}^{\prime}$. Then $R^{*}$ is a 2 -dimensional normal local domain [N, (33.10) and (33.12)], and $a, b$ form a regular sequence in $R^{*}$. Let $\phi^{*}: R^{*}[t] \rightarrow R^{*}[a / b]$ denote the $R^{*}$-algebra homomorphism such that $\phi^{*}(t)=a / b$. Then $\operatorname{ker} \phi^{*}=(b t-a) R^{*}[t] \subseteq \mathbf{m} R^{*}[t]$. Since $\phi^{*}$ restricts to $\phi$ on $D[t]$ and since $\mathbf{m} R^{*}[t] \cap D[t]=P[t]$, it follows that $\operatorname{ker} \phi \subseteq P[t]$.

Theorem 2.4. Let $(R, \mathbf{m})$ be a local domain and let $P$ be a prime ideal of $R$ such that $\operatorname{dim}(R / P)=d \geq 2$. There exists a prime ideal $Q$ of $R(t)$ such that

(1) $\operatorname{dim}(R(t) / Q)<d$,

(2) $Q \cap R=P$, and

(3) each P-primary ideal of $R$ is the contraction of a $Q$-primary ideal of $R(t)$.

Proof. Since $\operatorname{dim}(R / P) \geq 2$, we can choose $a \in \mathbf{m}-P$, and $b \in \mathbf{m}$ so that $b$ is not in any minimal prime of $(P, a) R$ nor in any minimal prime of $a R$. It follows that $\operatorname{ht}((a, b) R)=2$. Let $R^{\prime}=R / P$ and let $a^{\prime}, b^{\prime}$ denote the images of $a, b$ in $R^{\prime}$. Our choice of $a$ and $b$ implies that ht $\left(\left(a^{\prime}, b^{\prime}\right) R^{\prime}\right)=2$. Let $t$ be an indeterminate and consider the $R$-algebra homomorphism $\phi: R[t] \rightarrow R[a / b]$ such that $\phi(t)=a / b$, and the $R^{\prime}$-algebra homomorphism $\phi^{\prime}: R^{\prime}[t] \rightarrow R^{\prime}\left[a^{\prime} / b^{\prime}\right]$ such that $\phi^{\prime}(t)=a^{\prime} / b^{\prime}$. Since $b \notin P, R[a / b]$ is a subring of $R_{P}$ and $R_{P}=R[a / b]_{\mathbf{q}}$, where $\mathbf{q}=P R_{P} \cap R[a / b]$. By Lemma (2.3) and Remark (2.2), $\phi$ extends to a surjective $R$-algebra homomorphism $\psi: R(t) \rightarrow R[a / b]_{\mathbf{m} R[a / b]}$. We claim that

$$
\mathbf{q}=P R_{P} \cap R[a / b] \subseteq \mathbf{m} R[a / b] .
$$

Assuming (2.5), we have $R[a / b]_{\mathbf{m} R[a / b]} \subseteq R[a / b]_{\mathbf{q}}=R_{P}$. Let

$$
Q=\psi^{-1}\left(\mathbf{q} R[a / b]_{\mathbf{m} R[a / b]}\right) .
$$

Since $\psi(R(t)) \subseteq R_{P}$ and every $P$-primary ideal of $R$ is the contraction of a $P R_{P^{-}}$ primary ideal of $R_{P}$, it follows that every $P$-primary ideal of $R$ is the contraction of a $Q$-primary ideal of $R(t)$. Since $P R(t)<Q$ and $R(t) / P R(t) \cong(R / P)(t)$ is of dimension $d, \operatorname{dim}(R(t) / Q)<d$.

It remains to prove (2.5). Let $g: R_{P} \rightarrow R_{P} / P R_{P}$ denote the canonical surjection. Consider the following commutative diagram, where $f$ is the restriction of $g$, the map of $R[t]$ onto $R^{\prime}[t]$ is obtained by reducing coefficients modulo $P$, and $\alpha$ and 
$\beta$ are inclusion maps.

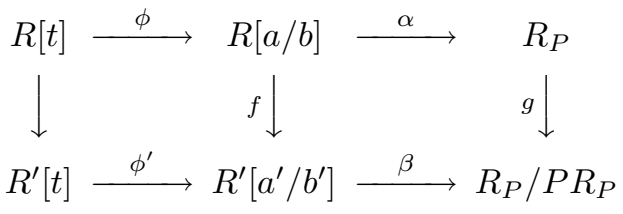

Let $\mathbf{m}^{\prime}=\mathbf{m} / P$ denote the maximal ideal of $R^{\prime}$. By Lemma $(2.3)$, ker $\phi^{\prime} \subseteq \mathbf{m}^{\prime}[t]$. By commutativity of (2.6), ker $f=\mathbf{q} \subseteq \mathbf{m} R[a / b]$. This establishes (2.5) and completes the proof of Theorem 2.4.

Corollary 2.7. Let $(R, \mathbf{m})$ be a local domain and let $I$ be an ideal of $R$ such that $\operatorname{dim}(R / I)=d \geq 2$. There exists an ideal $J$ of $R(t)$ such that $J \cap R=I$, every associated prime of $J$ in $R(t)$ contracts in $R$ to an associated prime of $I$, and $\operatorname{dim}(R(t) / J)<d$

Proof. Let $I=\bigcap_{i=1}^{n} \mathbf{w}_{i}$ be a minimal primary decomposition (cf. [AM, page 52]) of $I$ in $R$, where $\mathbf{w}_{i}$ is $P_{i}$-primary for $1 \leq i \leq n$. For each $i$ such that $\operatorname{dim}\left(R / P_{i}\right)=d$, Theorem 2.4 guarantees existence of a prime ideal $Q_{i}$ of $R(t)$ such that $\operatorname{dim}\left(R(t) / Q_{i}\right)$ $<d$ and such that there exists a $Q_{i}$-primary ideal $\mathbf{q}_{i}$ of $R(t)$ such that $\mathbf{q}_{i} \cap R=\mathbf{w}_{i}$. On the other hand, if $\operatorname{dim}\left(R / P_{i}\right)<d$, let $\mathbf{q}_{i}=\mathbf{w}_{i} R(t)$. In this latter case we have $R(t) / \mathbf{q}_{i} \cong\left(R / \mathbf{w}_{i}\right)(t)$, so $\operatorname{dim}\left(R(t) / \mathbf{q}_{i}\right)<d$. Let $J=\bigcap_{i=1}^{n} \mathbf{q}_{i}$. Then $\operatorname{dim}(R(t) / J)<d$ and $J \cap R=\bigcap_{i=1}^{n}\left(\mathbf{q}_{i} \cap R\right)=\bigcap_{i=1}^{n} \mathbf{w}_{i}=I$, so every associated prime of $J$ in $R(t)$ contracts in $R$ to an associated prime of $I$.

Theorem 2.8. Let $(R, \mathbf{m})$ be a local domain and let $I$ be an ideal of $R$ such that $\operatorname{dim}(R / I)=d \geq 1$. Let $t_{1}, \ldots, t_{d-1}$ be indeterminates over $R$ and let $S=$ $R\left(t_{1}, \ldots, t_{d-1}\right) .^{2}$ There exists an ideal $J$ of $S$ such that $J \cap R=I$, every associated prime of $J$ in $S$ contracts in $R$ to an associated prime of $I$, and $\operatorname{dim}(S / J)=1$.

Proof. We proceed by induction on $d=\operatorname{dim}(R / I)$. If $d=1$, the assertion is clear. Assume $\operatorname{dim}(R / I)=d \geq 2$ and that the assertion holds for all local domains $T$ and ideals $L$ of $T$ such that $1 \leq \operatorname{dim}(T / L)<d$. By $(2.7)$, there exists an ideal $J$ of $R\left(t_{1}\right)$ such that $J \cap R=I$, every associated prime of $J$ in $R\left(t_{1}\right)$ contracts in $R$ to an associated prime of $I$, and $\operatorname{dim}\left(R\left(t_{1}\right) / J\right)<d$. Since a zero-dimensional local ring has nilpotent maximal ideal and therefore cannot dominate a local ring of positive dimension, we have $\operatorname{dim}\left(R\left(t_{1}\right) / J\right) \geq 1$. Applying the induction hypothesis to the ideal $J$ of the ring $R\left(t_{1}\right)$ now yields the result.

We summarize our results of this section in the following corollary.

Corollary 2.9. Let $(R, \mathbf{m})$ be a local domain and let $I$ be an ideal of $R$ such that $\operatorname{dim}(R / I)=d \geq 1$. Let $t_{1}, \ldots, t_{d-1}$ be indeterminates over $R$ and let $S=$ $R\left(t_{1}, \ldots, t_{d-1}\right) .{ }^{3}$ Then $S$ is a local extension domain of $R$ such that:

(1) $\mathbf{m} S$ is the maximal ideal of $S$,

(2) $S / \mathbf{m} S$ is a purely transcendental extension field of $R / \mathbf{m}$ of transcendence degree $d-1$, and

(3) there exists an ideal $J$ of $S$ such that $J \cap R=I$, every associated prime of $J$ in $S$ contracts in $R$ to an associated prime of $I$, and $\operatorname{dim}(S / J)=1$.

\footnotetext{
${ }^{2}$ If $d=1$, we take $S=R$.

${ }^{3}$ Again, if $d=1$, we take $S=R$.
} 
Proof. The first two assertions about $S$ are noted in (2.1), and the third condition is established in (2.8).

\section{Domination By A OnE-Dimensional LOCAL RING}

As a first application of Theorem 2.8, we have:

Theorem 3.1. Let $(R, \mathbf{m})$ be a local ring with $\operatorname{dim}(R)=d \geq 1$. There exists a local extension ring $S$ of $R$ such that:

(1) $\mathbf{m} S$ is the maximal ideal of $S$,

(2) $S / \mathbf{m} S$ is a pure transcendental extension field of $R / \mathbf{m}$ in $d-1$ indeterminates,

(3) every associated prime of (0) in $S$ contracts in $R$ to an associated prime of (0) in $R$, so regular elements of $R$ remain regular in $S$, and

(4) $\operatorname{dim}(S)=1$.

Proof. Let $\widehat{R}$ denote the completion of $R$ with respect to the $\mathbf{m}$-adic topology. Then $\widehat{R}$ is a local extension ring of $R$ with maximal ideal $\mathbf{m} \widehat{R}$, every associated prime of (0) in $\widehat{R}$ contracts in $R$ to an associated prime of (0) in $R$, (cf. [N, (18.11)]), and $R / \mathbf{m}=\widehat{R} / \mathbf{m} \widehat{R}$ with respect to the canonical inclusion of $R / \mathbf{m}$ into $\widehat{R} / \mathbf{m} \widehat{R}$. A structure theorem of Cohen [C, Theorem 12],[N, (31.1)] implies the existence of a complete local domain $(T, \mathbf{n})$ having an ideal $I$ such that $T / I \cong \widehat{R}$. By Corollary 2.9, there exists a local extension domain $W=T\left(t_{1}, \ldots, t_{d-1}\right)$ of $T$ such that: (i) $\mathbf{n} W$ is the maximal ideal of $W$, (ii) $W / \mathbf{n} W$ is a pure transcendental extension of $T / \mathbf{n}$ in $d-1$ indeterminates, (iii) there exists an ideal $J$ of $W$ such that $J \cap T=I$, every associated prime of $\mathrm{J}$ in $W$ contracts in $T$ to an associated prime of $I$, and (iv) $\operatorname{dim}(W / J)=1$. We have $R \hookrightarrow \widehat{R} \cong T / I \hookrightarrow W / J$. Therefore $S=W / J$ is a local extension of $R$ of the desired form.

An alternate proof of parts of Theorem 3.1 can be obtained by using the concept of gluing of maximal ideals. This gluing process has the merit of yielding a onedimensional local extension ring $S$ dominating $R$ which is a localization of a finitely generated $R$-algebra.

The following lemma extends (2.3) to a ring context.

Lemma 3.2. Let $E$ be a Noetherian ring such that (0) in $E$ is $\mathbf{p}$-primary. Suppose $a, b \in E$ are such that $\mathrm{ht}((a, b) E)=2$ and let $P$ be a prime ideal of $E$ such that $(a, b) E \subseteq P$. Since $\operatorname{ht}((a, b) E)=2, b \notin \mathbf{p}$, so $E[a / b] \subseteq E_{\mathbf{p}}$, the total quotient ring of $E$. Let $t$ be an indeterminate over $E$ and let $\phi: E[t] \rightarrow E[a / b]$ be the E-algebra homomorphism such that $\phi(t)=a / b$. Then $\operatorname{ker} \phi \subseteq P[t]$.

Proof. Since $E[a / b] \subseteq E_{\mathbf{p}}$, the ideal (0) of $E[a / b]$ is primary and $\mathbf{p} E_{\mathbf{p}} \cap E[a / b]=\mathbf{q}$ is the nilradical of $E[a / b]$. Let $a^{\prime}, b^{\prime}$ denote the images of $a, b$ in $D=E / \mathbf{p}$. Consider the following commutative diagram:

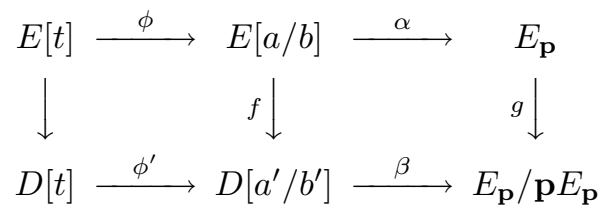

where $\phi^{\prime}: D[t] \rightarrow D\left[a^{\prime} / b^{\prime}\right]$ is the $D$-algebra homomorphism such that $\phi^{\prime}(t)=a^{\prime} / b^{\prime}$, the map $E[t] \rightarrow D[t]$ is reduction of coefficients modulo $\mathbf{p}, g: E_{\mathbf{p}} \rightarrow E_{\mathbf{p}} / \mathbf{p} E_{\mathbf{p}}$ is the 
canonical surjection, $f$ is the restriction of $g$, and $\alpha$ and $\beta$ are inclusion maps. By Lemma 2.3, $\operatorname{ker} \phi^{\prime} \subseteq(P / \mathbf{p}) D[t]$. Hence $\operatorname{ker} \phi \subseteq P[t]$.

Theorem 3.4. Let $(R, \mathbf{m})$ be a local ring such that $(0)$ in $R$ is $\mathbf{p}$-primary. Assume that $\operatorname{dim}(R)=d \geq 2$. There exists a local extension ring $T$ of $R$ such that

(1) $T$ is a localization of a finitely generated $R$-algebra,

(2) $\mathbf{m} T$ is the maximal ideal of $T$ so that, in particular, $T$ dominates $R$,

(3) $T$ is a subring of the total quotient ring of $R$, so the ideal (0) of $T$ is primary,

(4) $\operatorname{dim}(T)=1$, and

(5) there exists a positive integer $n \leq d-1$ such that the residue field of $T$ is canonically isomorphic to $(R / \mathbf{m})\left(t_{1}, \ldots, t_{n}\right)$, a pure transcendental extension of $R / \mathbf{m}$ in $n$ indeterminates.

Proof. Since $d \geq 2$ there exist $a, b \in \mathbf{m}$ such that $\mathrm{ht}((a, b) R)=2$. It follows that $b \notin \mathbf{p}$ and hence that $b$ is a regular element of $R$, so $R[a / b]$ is a subring of the total quotient ring $R_{\mathbf{p}}$ of $R$. Let $\phi: R[t] \rightarrow R[a / b] \subseteq R_{\mathbf{p}}$ be the $R$-algebra homomorphism such that $\phi(t)=a / b$. Lemma 3.2 implies that $\operatorname{ker} \phi \subseteq \mathbf{m}[t]$. By (2.2), $\phi$ extends to a surjective $R$-algebra homomorphism of $R(t)$ onto $R[a / b]_{\mathbf{m} R[a / b]}$ and $R(t) /(\operatorname{ker} \phi) R(t) \cong R[a / b]_{\mathbf{m} R[a / b]}$. It is clear that $S=R[a / b]_{\mathbf{m} R[a / b]}$ is a local ring with maximal ideal $\mathbf{m} S$. The ideal $(0)$ of $S$ is primary since the zero ideal of $R_{\mathbf{p}}$, the total quotient ring of $S$, is primary. Each of the local rings $R(t)$ and $S$ has residue field isomorphic to $(R / \mathbf{m})(t)$. The polynomial $b t-a$ is an element of $(\operatorname{ker} \phi) R(t)$ that is not in $\mathbf{p} R(t)$, the unique minimal prime of $R(t)$. Hence $S \cong R(t) /(\operatorname{ker} \phi) R(t)$ has dimension less than $\operatorname{dim}(R(t) / \mathbf{p} R(t))=d$. Since $\mathbf{m}$ is not nilpotent, $\mathbf{m} S$ is not nilpotent, so $\operatorname{dim}(S)>0$. The $\operatorname{ring}(S, \mathbf{m} S)$ satisfies the hypothesis of Theorem 3.4, so by repetition of the process above if necessary (that is, if $\operatorname{dim}(S)>1$ ), we obtain after at most $\operatorname{dim}(S)-1$ repetitions a one-dimensional local ring $T$ which satisfies the conditions of Theorem 3.4

(3.5). Suppose $(S, \mathbf{n})$ is a one-dimensional local ring in which (0) is primary and $t_{1}, \ldots, t_{k}$ are indeterminates over $S$. Then $S\left(t_{1}, \ldots, t_{k}\right)$ is a one-dimensional local ring with maximal ideal $\mathbf{n} S\left(t_{1}, \ldots, t_{k}\right)$ and residue field $(S / \mathbf{n})\left(t_{1}, \ldots, t_{k}\right)$, a pure transcendental extension of $S / \mathbf{n}$ in $k$ indeterminates; moreover, the zero ideal of $S\left(t_{1}, \ldots, t_{k}\right)$ is primary. Therefore with notation as in Theorem 3.4 , by passing from $T$ to $W=T\left(t_{1}, \ldots, t_{k}\right)$, one obtains a local ring $W$ satisfying all the assertions in (3.4) except that $W$ is not a subring of the total quotient ring of $R$. Thus for each positive integer $s \geq d-1$, there exists a one-dimensional local extension ring $W_{s}$ of $R$ such that the residue field of $W_{s}$ is canonically isomorphic to $(R / \mathbf{m})\left(t_{1}, \ldots, t_{s}\right)$, a pure transcendental extension of $R / \mathbf{m}$ in $s$ indeterminates, and such that $W_{s}$ satisfies all the conditions of (3.4) except that of being a subring of the total quotient ring of $R$.

(3.6). The process of gluing maximal ideals proceeds as follows; the reader is referred to [DL] for details. Assume that $M_{1}, \ldots, M_{k}$ are maximal ideals of a ring $T$ such that each $T / M_{i}$ is isomorphic to a field $F$. Suppose $\phi_{i}: T \rightarrow F$ is a surjective ring homomorphism such that $\operatorname{ker} \phi_{i}=M_{i}$ for $1 \leq i \leq k$. Let

$$
S=\left\{t \in T: \phi_{1}(t)=\phi_{2}(t)=\cdots=\phi_{k}(t)\right\} .
$$

Then $S$ is a subring of $T$ containing $M=M_{1} \cap \cdots \cap M_{k}$ as a maximal ideal, $S / M \cong F, T$ is a finitely generated integral extension of $S$, and each of the maximal ideals $M_{i}$ lies over $M$ in $S$. We say that $S$ is a gluing of the maximal ideals 
$M_{1}, \ldots, M_{k}$. If the ring $T$ is Noetherian, then by Eakin's theorem [M, page 18], $S$ is Noetherian. Since $S$ is a subring of $T$, each associated prime of (0) in $S$ is the contraction to $S$ of an associated prime of (0) in $T$.

Theorem 3.7. Let $(R, \mathbf{m})$ be a local ring. There exists a local extension ring $S$ of $R$ such that

(1) $S$ dominates $R$,

(2) the residue field of $S$ is of the form $(R / \mathbf{m})\left(t_{1}, \ldots, t_{k}\right)$, a pure transcendental extension of $R / \mathbf{m}$,

(3) $S$ is a localization of a finitely generated $R$-algebra,

(4) every associated prime of (0) in $S$ contracts in $R$ to an associated prime of $(0)$, so every regular element of $R$ remains regular in $S$, and

(5) $\operatorname{dim}(S) \leq 1$, and $\operatorname{dim}(S)=1$ unless $\operatorname{dim}(R)=0$.

Proof. Let $d=\operatorname{dim}(R)$. If $d \leq 1$, then for any positive integer $k, S=R\left(t_{1}, \ldots, t_{k}\right)$ satisfies the required conditions.

If $d \geq 2$, let (0) $=\bigcap_{i=1}^{n} \mathbf{q}_{i}$ be a minimal primary decomposition of (0) in $R$ and let $R_{i}=R / \mathbf{q}_{i}$. Then $R_{i}$ is a local ring with maximal ideal $\mathbf{m}_{i}=\mathbf{m} / \mathbf{q}_{i}, \operatorname{dim}\left(R_{j}\right) \leq d$ for each $j$, and $\operatorname{dim}\left(R_{j}\right)=d$ for at least one integer $j, 1 \leq j \leq n$. By (3.4) and (3.5), there exist local rings $\left(T_{i}, M_{i}\right), 1 \leq i \leq n$, such that $T_{i}$ is the localization of a finitely generated $R_{i}$-algebra, the ideal (0) of $T_{i}$ is primary, $\mathbf{m}_{i} T_{i}$ is the maximal ideal of $T_{i}$, $\operatorname{dim}\left(T_{i}\right) \leq 1$, and each $T_{i} / \mathbf{m}_{i} T_{i}$ is canonically isomorphic to $(R / \mathbf{m})\left(t_{1}, \ldots, t_{k}\right)$ for some positive integer $k$; moreover, $\operatorname{dim}\left(T_{j}\right)=1$ for some $j$, and hence $\operatorname{dim}(T)=1$. The ring $T=T_{1} \oplus \cdots \oplus T_{n}$ is Noetherian with $n$ maximal ideals $N_{1}, \ldots, N_{n}$ where, for $1 \leq i \leq n, T / N_{i} \cong T_{i} / \mathbf{m}_{i} T_{i} \cong(R / \mathbf{m})\left(t_{1}, \ldots, t_{k}\right)$. Moreover, $T$ has $n$ minimal primes and every associated prime of $(0)$ in $T$ is a minimal prime. We identify $R$ as a subring of $T$ via the diagonal embedding $r \mapsto\left(r+\mathbf{q}_{1}, \ldots, r+\mathbf{q}_{n}\right)$. Let $\phi_{i}$ denote the canonical surjection of $T$ onto $(R / \mathbf{m})\left(t_{1}, \ldots, t_{k}\right)$ such that $\operatorname{ker} \phi_{i}=N_{i}$, and let $S$ denote the gluing of $N_{1}, \ldots, N_{n}$ with respect to $\phi_{1}, \ldots, \phi_{n}$. Then $R$ is a subring of $S$. Each associated prime of (0) in $S$ is the contraction to $S$ of an associated prime of (0) in $T$, and therefore contracts in $R$ to an associated prime of (0). Moreover, $\mathbf{n}=N_{1} \cap \cdots \cap N_{n}$ is the unique maximal ideal of $S$. The ring $S$ is Noetherian since $T$ is a Noetherian ring and $T$ is a finitely generated $S$-module. Because $T_{i}$ is a localization of a finitely generated $R_{i}$-algebra for each $i$, it follows easily that the ring $T$ is a localization of a finitely generated extension ring of $R$ in $T$. Consequently, $S$ is a localization of a finitely generated $R$-algebra [HL, Prop. 1.1, page 2867]. We have $\operatorname{dim}(S)=\operatorname{dim}(T)=1$, so $S$ is a one-dimensional local ring.

Remark 3.8. The gluing construction in the proof of (3.7) provides an $S$ so that $\mathbf{m} S$ is primary for the maximal $\mathbf{n}$ of $S$, but, in general, not with $\mathbf{m} S=\mathbf{n}$. With notation as in the proof of (3.7), we have

$$
\mathbf{m} T=\mathbf{m}_{1} T_{1} \oplus \cdots \oplus \mathbf{m}_{n} T_{n}=M_{1} \oplus \cdots \oplus M_{n},
$$

and the projection of $\mathbf{m} S$ onto $T_{i}$ is $M_{i}=\mathbf{m}_{i} T_{i}$ for each $i$. But the kernels of these projection maps on $T$ are not contained in $S$, and one may have $\mathbf{m} S<\mathbf{n}=\mathbf{m} T$. In the case where $R$ has only one associated prime of (0), Theorem 3.4 implies the existence of an extension $S$ of $R$ satisfying both the condition in (3.1) that $\mathbf{m} S$ is the maximal ideal of $S$ and the condition of (3.7) that $S$ is a localization of a finitely generated $R$-algebra. It would be interesting to know more generally whether there exists an $S$ simultaneously satisfying the conditions of (3.1) and (3.7). 


\section{REFERENCES}

[AM] M. Atiyah and I. Macdonald, Introduction to Commutative Algebra, Addison-Wesley, Reading, MA, 1969. MR 39:4129

[CHL] P.-J. Cahen, E. G. Houston, and T. G. Lucas, Discrete valuation overrings of Noetherian domains, Proc. Amer. Math. Soc. 124 (1996), 1719-1721. MR 96h:13057

[Ch] C. Chevalley, La notion d'anneau de dećomposition, Nagoya Math. J. 7 (1954), 21-33. MR 16:788g

[C] I. S. Cohen, On the structure and ideal theory of complete local rings, Trans. Amer. Math. Soc. 59 (1946), 54-106. MR 7:509h

[DL] A.M.S. Doering and Y. Lequain, The gluing of maximal ideals - spectrum of a Noetherian ring - going up and going down in polynomial rings, Trans. Amer. Math. Soc. 260 (1980), 583-593. MR 81g:13014

[G] R. Gilmer, Multiplicative Ideal Theory, Queen's Papers Pure Appl. Math. Vol 90, Kingston, 1992. MR 93j: 13001

[GH1] R. Gilmer and W. Heinzer, The Noetherian property for quotient rings of infinite polynomial rings, Proc. Amer. Math. Soc. 76 (1979), 1-7. MR 80h:13010

[GH2] R. Gilmer and W. Heinzer, Ideals contracted from a Noetherian extension ring, J. Pure Appl. Algebra 24 (1982), 123-144. MR 84a:13006

[GH3] R. Gilmer and W. Heinzer, The family of residue fields of a zero-dimensional commutative ring, J. Pure Appl. Algebra 82 (1992), 131-153. MR 93k:13019

[GH4] R. Gilmer and W. Heinzer, Artinian subrings of a commutative ring, Trans. Amer. Math. Soc. 336 (1993), 295-310. MR 93e: 13028

[GH5] R. Gilmer and W. Heinzer, Imbeddability of a commutative ring in a finite-dimensional ring, Manuscripta Math. 84 (1994), 401-414. MR 95i:13004

[HL] W. Heinzer and D. Lantz, Ideal theory in two-dimensional regular local domains and birational extensions, Comm. in Algebra 23 (1995), 2863-2880. MR 96d:13037

[H] J. Huckaba, Commutative Rings with Zero Divisors, Marcel-Dekker, New York, 1988. MR 89e:13001

[M] H. Matsumura, Commutative Ring Theory, Cambridge University Press, 1986. MR 88h:13001

[N] M. Nagata, Local Rings, Interscience, 1962. MR 27:5790

Department of Mathematics, Florida State University, Tallahassee, Florida 32306 3027

E-mail address: gilmer@math.fsu.edu

Department of Mathematics, Purdue University, West Lafayette, Indiana 47907-1395

E-mail address: heinzer@math.purdue.edu 